\title{
Characterizing the Nature of Human Carriers of Head Lice
}

\author{
Deon V. Canyon*, Chauncey Canyon and Sami Milani
}

Office of Public Health Studies, University of Hawaii at Manoa, 1960 East-West Rd, Biomed Building \#T103, Honolulu HI 96822, USA

\begin{abstract}
Pediculosis is a ubiquitous disease common throughout the globe and managed entirely through the application of insecticides and natural therapies with varying success. Resistance and reinfection are known to be responsible for increases in prevalence reported in many countries since the 1900s. This study investigated reinfection, which has been neglected by researchers, by attempting to learn more about the role of reservoir hosts. Open-ended questions were asked from 126 students of Grades 8 and 9 to explore this issue from the perspective of the infected population. A majority of females $(60 \%)$ and $40 \%$ of males had no idea that they had pediculosis until it was discovered by themselves, a friend or a carer. Some female (12.1\%) and male (14.5\%) students did not tell their parents when they became aware that they had pediculosis. Hair thickness was significantly related to this question with $23.3 \%$ of thick-haired students concealing their infections. The results from this study suggest that brown- and short-haired White boys should be a primary target in lice awareness and control programs, because they are much more likely to harbor lice than long-haired girls. Health professionals should be aware of the social reasons for why this pest is so difficult to control.
\end{abstract}

Keywords: Carrier, head lice, pediculosis, reservoir, transmission.

\section{INTRODUCTION}

Pediculosis is a classic global health disease since it affects all people in all nations, crosses national boundaries and most countries have experienced increases in the detection of Pediculus humanus var. capitis (De Geer) since the 1900s [1]. Head lice are obligate human parasites that have coexisted with humans for at least 9000 years [2]. Physical, botanical and chemical methods have been used to control head lice since earliest recorded history with limited success.

While reducing transmission is fundamental to control the spread of any infection, pediculosis often eludes management efforts and the way forward is not clear when treatment fails. The persistence of head lice in modern society has often been attributed to lack of hygiene, a mobile population and insecticide resistance while the underlying social aspects of head lice transmission have received far less attention. Despite multiple treatments with effective pediculicides, lice may continually return to cause great alarm to parents that exceeds their medical significance [3,4]. Reinfection is a highly problematic and understudied social issue that merits further consideration and study $[5,6]$. Is it due to an unfortunate chance meeting of an uninfected child with an infected child or are there patterns of social behavior that enable head lice populations to persist in a population? Do these behaviors have something to do with the newfound role of feelings and emotions related to head lice infection [7-9]? These questions have remained unexplored by researchers, so this study sought to shed light on the nature of human head lice carriers.

*Address correspondence to this author at the Office of Public Health Studies, University of Hawaii at Manoa, 1960 East-West Rd, Biomed Building \#T103, Honolulu HI 96822, USA; Tel: (808) 956-6263; E-mail: dcanyon@hawaii.edu

\section{METHODS}

The site for this study was a multicultural, middle-class, public school in Perth, Western Australia, which was one of the top performing schools in the state. The student body totaled around 1700 students in Grades 8 to 12 with 480 middle-school students in Grades 8 and 9. Participants for this study were sought from the middle school and information on the study was provided to parents who completed the consent form. Teenagers with parental consent were asked to complete an anonymous survey, which they deposited in a box in the school front office. This retrospective study requested information on pediculosis incidence based on student recall. In Australia, primary schools, in which head lice infections predominantly occur, run from Grades 1 to 7. Completed consent forms and surveys were collected from the school for analysis. Participation was thus voluntary and self-selecting. Only students who could recall being infected with head lice were included in the study. This sampling strategy combines criterion based and convenience approaches, since the targeted participants are those who have direct experience coping with a head lice infestation (criterion) and they are accessible (convenience) [10].

The questionnaire requested basic non-identifying demographic information (grade, gender and hair details) followed by three open-ended questions:

1. Have you ever been surprised to find out that you had head lice when you did not know that you had them?

2. Have you ever known you had head lice and didn't tell your parents?

3. If you had lice and you did not tell your parents, why didn't you tell them? 
The anonymous responses were analyzed using the computer software package SPSS 21. Cross-tabulations with Chi-Square tests were employed, but since frequencies were occasionally less than five per cell, this test was replaced with the Uncertainty Coefficient analysis. Ethics approval H2954 was obtained from James Cook University.

\section{RESULTS}

Out of 480 students contacted, 133 confirmed a history of head lice infection, volunteered to participate and submitted study surveys with a response rate $27.7 \%$. Six surveys were discarded due to being too incomplete for analysis, leaving 127 participant surveys for the study (Table 1). The temporal proximity of participants to the infection event and the nature of the event being recalled indicate that recall would have been reasonably accurate. While adult retrospective reports of adverse childhood experiences involve a substantial rate of false negatives, false positive reports are rare [11].

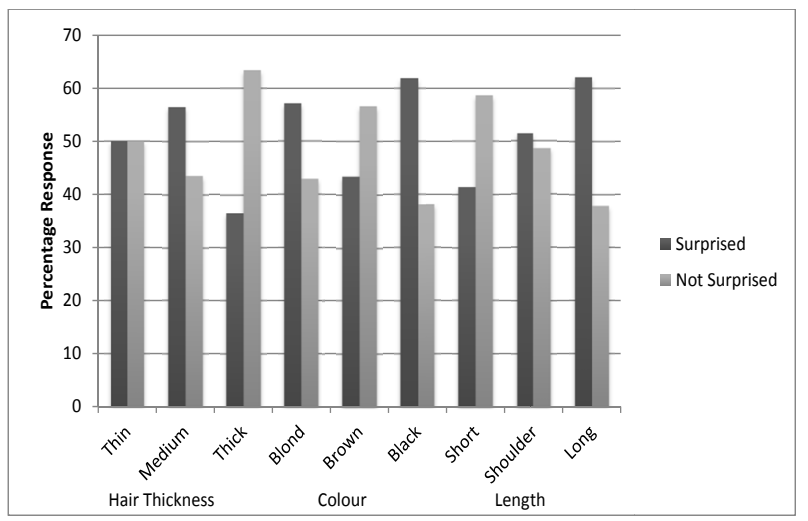

Fig. (2). The relationship between hair thickness, colour and length in participants who were unsurprised and surprised to find out they were infected with head lice.

Six female and ten male students concealed their infection from their parents and there was no significant gender effect. All

Table 1. Demographic data for the sample.

\begin{tabular}{|c|c|c|c|c|c|c|}
\hline Grade & Females (\%) & Males (\%) & Missing & Caucasians (\%) & Asians (\%) & Missing (\%) \\
\hline \hline 8 & $28(41.2)$ & $38(55.9)$ & $2(2.9)$ & $59(86.8)$ & $8(11.9)$ & $1(1.5)$ \\
\hline 9 & $30(50.8)$ & $29(49.2)$ & $0(0)$ & $49(83.1)$ & $9(15.3)$ & $1(1.7)$ \\
\hline
\end{tabular}

In response to the question, "Have you ever been surprised to find out that you had head lice when you did not know that you had them?" males were less likely than females to be surprised that they had a head lice infection. Pearson's ChiSquare analysis showed that the relationship between the responses to the question and gender was significant (4.310, $\mathrm{p}<0.05$ ), but that ethnicity was not. Nevertheless, there were distinct ethnic differences observable in Fig. (1).

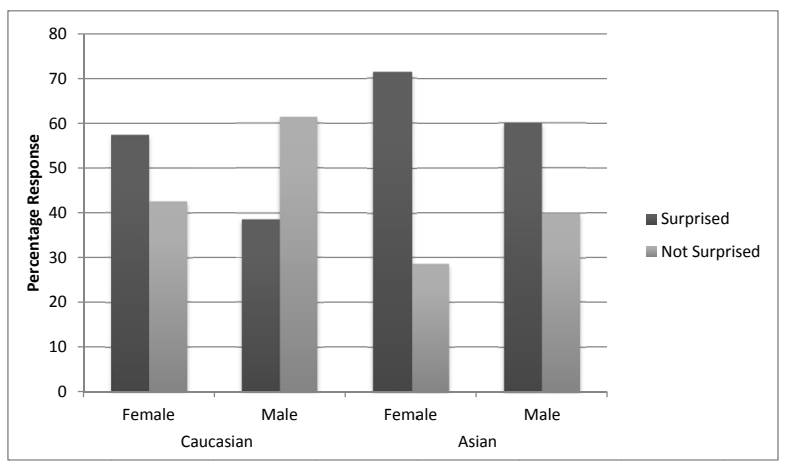

Fig. (1). The percentage of Caucasian and Asian females and males that were surprised or not surprised to find out they were infected with head lice.

Hair thickness, color and length were not significantly associated with surprise in this analysis $(p>0.05)$. However, Fig. (2) shows that respondents with short brown thick hair were more often not surprised to find that they had pediculosis. The most surprised were those with medium thickness blond or black long hair.

Participants were then asked if they had ever known they had head lice and had concealed the infection from their parents. those who concealed their infection were Caucasian. Uncertainty coefficient analysis showed that the relationship between the responses to the question and ethnicity was significant $(\mathrm{p}<0.05)$, although the relationship was weak $(\mathrm{u}=0.052)$. This was reflected in the hair color result which showed that all black-haired students told their parents and $17.9 \%$ of brown-haired students did not tell their parents (Chi square $6.264, \mathrm{p}<0.05$ ). Only one blond haired student concealed an infection. Uncertainty coefficient analysis also showed a trend in the relationship between the responses to the question and hair thickness (Chi square 5.475, $\mathrm{p}=0.066$ ), with thickhaired students being twice as likely as medium-haired students to conceal an infection from their parents (Fig. 3).

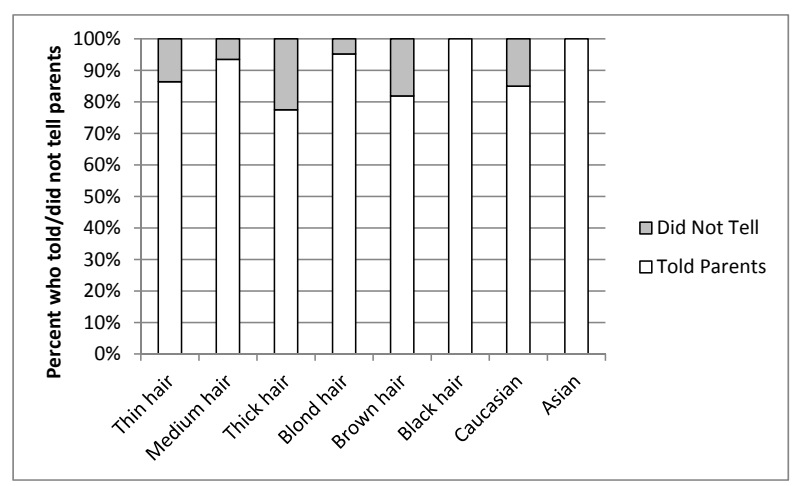

Fig. (3). Percentage of students who concealed a head lice infection from their parents compared with hair thickness and color.

When asked the third question, "If you had lice and you did not tell your parents, why didn't you tell them?" twelve males and nine females stated reasons. Five answered that they did not want their parents to get annoyed, angry or have problems; three 
were embarrassed or ashamed; five did not want to be treated; and eight did not know the reason. None of the study factors were significantly related to this question. Uncertainty coefficient analysis revealed a moderate trend $(p=0.77$, $\mathrm{u}=0.111$ ) for thick-haired students who did not want to have problems with their parents.

\section{DISCUSSION}

The aim of this study was to shed light on the nature of human head lice carriers and describe the extent to which children conceal head lice infection from their parents. Concealment is an important prevalence and transmission variable because it results in individuals becoming carriers, which invariably increases the availability of head lice in a community. Concealment is thought to derive mainly from social and emotional feelings that influence the behavior of infected individuals and their carers [7-9]. The results from this study provided evidence to suggest that brown-haired, shorthaired boys should be a primary target in lice awareness and control programs, but that other people should not be overlooked.

Results from the question, "Have you ever been surprised to find out that you had head lice when you did not know that you had them?" indicated that females were more likely to be surprised than males upon discovering that they have pediculosis. The important point here is that $60 \%$ of females and $40 \%$ of males had no idea that they had pediculosis until it was discovered. This means that they may act as head lice carriers and transmitters for a considerable amount of time until the infection becomes noticed by themselves or someone else. Hair length is a likely explanation because head lice infections are harder to detect on people with longer hair.

In response to the very direct question, "Have you ever known you had head lice and didn't tell your parents?" $10.7 \%$ of female and $14.7 \%$ of male students admitted to not telling their parents when they became aware that they had pediculosis. Hair thickness was significantly related to this question with $22.0 \%$ of thick-haired students concealing their infections. It may be that combing with a fine-toothed comb would be more painful for thick-haired people, which gives them a practical reason for concealing infection. The diameter of hairs on the human head varies between individuals from $0.017 \mathrm{~mm}$ to $0.181 \mathrm{~mm}$ [12]. Fine, medium and coarse hair are generally classified as $<0.05,0.06$ to 0.09 , and $>0.1 \mathrm{~mm}$ wide, respectively. The most popular head lice comb is the Lice Meister which has metal prongs spaced $0.094 \mathrm{~mm}$ wide and splaying reduces this to almost zero at the base of the prongs. The comb would prove painful and would remove many hairs upon use.

The common public perception is that longhaired females who do a lot of head-to-head socializing are primary head lice carriers and transmitters. By questioning teenagers, this study gained considerable insight which demonstrated that this perception is incorrect. Of those teenagers who were aware of their head lice infections, $64.5 \%$ were males and 35.5\% were females; of those who did not inform their parents when they knew that they had lice, $53.7 \%$ were males and $46.3 \%$ were females; of those who did not tell their parents because they did not want to be treated, $80 \%$ were males and $20 \%$ were females; and of those who could not explain why they did not tell their parents or thought the infection was not important, 57.1\% were males and $42.9 \%$ were females. Maunder (1985) had a healthy suspicion when he stated that attitude to head lice is a more powerful force than insecticides [13]. If there is to be any generalized perception of who carries head lice in a western multicultural setting, it is that boys with short brown hair should be suspected well before longhaired girls.

\section{CONFLICT OF INTEREST}

The authors confirm that this article content has no conflict of interest.

\section{ACKNOWLEDGEMENTS}

\author{
Declared none.
}

\section{REFERENCES}

[1] Gratz NG. Human lice: their prevalence, control and resistance to insecticides: a review 1985-1997. Geneva: World Health Organization, Division of Control of Tropical Diseases, WHO Pesticide Evaluation Scheme, 1997.

[2] Mumcuoglu KY, Zias J. Head lice, Pediculus humanus capitis (Anoplura: Pediculidae), from hair combs excavated in Israel and dated from the first century B.C. to the eighth century A.D. J Med Entomol 1988; 25: 545-7.

[3] Counahan M. Scratching for answers? Public health aspects of head lice control. PhD thesis, Townsville, Qld: James Cook University 2006.

[4] Falagas M, Matthaiou DK, Petros IP, Panos G, Pappas G. Worldwide prevalence of head lice. Emerg Infect Dis 2008; 14: 1493-4.

[5] Canyon DV, Speare R. Clinical decision support: Dermatology: Pediculosis. Wilmington, DE: Decision Support in Medicine LLC, 2012.

[6] Canyon DV, Speare R. Head lice transmission and risk factors. In: Heukelbach J, Ed. Management and control of head lice infestations. Bremen: UNI-MED Verlag AG, 2010; 34-40.

[7] Parison J, Canyon DV. Head lice and the impact of knowledge, attitudes and practices - a social science overview. In: Heukelbach J, Ed. Management and control of head lice infestations. Bremen: UNIMED Verlag AG, 2010; 103-9.

[8] Parison J, Speare R, Canyon DV. Head lice: The feelings people have Int J Dermatol 2013; 52(2): 169-71.

[9] Parison J, Speare, R, Canyon DV. Uncovering family experiences with head lice: The difficulties of eradication. Open Dermatol J 2008; 2: 917.

[10] Patton MQ. Qualitative research \& evaluation methods, $3^{\text {rd }}$ ed Thousand Oaks, CA: Sage 2002.

[11] Hardt J, Rutter M. Validity of adult retrospective reports of adverse childhood experiences: Review of the evidence. J Child Psychol Psych 2004; 45(2): 260-73.

[12] Ley B. Diameter of a human hair; 1999; http://hypertextbook.com/facts/ 1999/BrianLey.shtml [Accessed: Apr 2014].

[13] Maunder B. Attitude to head lice--a more powerful force than insecticides. J R Soc Health 1985; 105(2): 61-4. 